\title{
Research on High-Frequency Asymmetric Pulse Power Supply of Current Source
}

\author{
Huang Xiping \\ Department of Automation and Information Engineering \\ Xi'an University of Technology \\ Shaanxi, P.R.China \\ huangxp@xaut.edu.cn
}

Sun Qiang

Department of Automation and Information Engineering

Xi'an University of Technology

Shaanxi, P.R.China

sq@xaut.edu.cn

\begin{abstract}
In order to meet the requirements of the surface treatment of material, in this paper the $50 \mathrm{kHz}$ asymmetrical pulse power supply of current source is developed. The power supply adopts a two-stage construction. The front stage, in which current closed-loop control is employed, is high-frequency isolated DC/DC converter. The latter stage, on the contrary, is non-isolated asymmetric high-frequency pulse generator and uses open-loop control. After analyzing the topological principle, the important effect of the methods of series LC filter in the front stage is discussed. The $1 \mathrm{~kW}$ experimental prototype is built and relevant experiments are carried out. Theoretical analysis and experimental results show that the circuit can meet the requirements of the surface treatment of material, and the circuit has the advantages of simple structure, easy control and low cost.
\end{abstract}

Keywords- current source; asymmetric pulse power supply; higfrequency switching-mode power supply

\section{INTRODUCTION}

Pulse power supply, especially the asymmetric pulse power in which a small amplitude negative pulse is superposed on a positive forward pulse, is widely used in all kinds of material surface treatment equipment. This paper studies a new highfrequency asymmetric pulse power of current source that adopts a two-stage structure. The front stage, a high-frequency $\mathrm{DC} / \mathrm{DC}$ converter of constant-current control, is used to realize positive pulse amplitude adjustment. The latter stage is an asymmetric pulse converter of which the frequency varies from $20 \mathrm{kHz}$ to $50 \mathrm{kHz}$ and the dutycycle ranges from $10 \%$ to $80 \%$. Under the circumstances of the load of resistive and inductive load, the output is the asymmetric voltage pulse. The pulse amplitude can change along of the load, which is very suitable for the requirements of surface treatment technology

\author{
Chen Guitao \\ Department of Automation and Information Engineering \\ Xi'an University of Technology \\ Shaanxi, P.R.China \\ cgtao@xaut.edu.cn
}

\author{
Shen Degui \\ Department of Automation and Information Engineering \\ Xi'an University of Technology \\ Shaanxi, P.R.China
}

of materials like magnetron sputtering and so on. After analyzing the topological principle, an experimental prototype was built. Based on the theoretical analysis and experimental results, it is concluded that the circuit can meet the requirements of the surface treatment of material, and the converter has a good application prospect.

\section{THE HIGN-FREQUENCY ASYMMETRIC PULSE POWER OF CURRENT SOURCE}

In order to reduce the volume and cost of power , and improve its efficiency of power supply, two-stage structure is used, the front stage is DC/DC high-frequency converter and the latter stage is asymmetric pulse converter. Below is an overall block diagram of the converter ${ }^{[1]}$ :

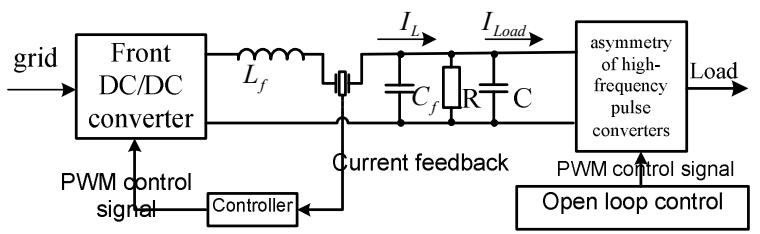

Figure 1. Overall block diagram of the high-frequency asymmetric pulse power of current source

Adding a capacitor $\mathrm{C}$ and a large resistor $\mathrm{R}$ to the latter stage's input portions, the asymmetric pulse amplitude can be controlled by regulating the frequency and duty cycle of the latter stage. Generally, the capacitor is a small high-frequency no-sense capacitor.The high-frequency components of the input current can be filtered so that the output pulse will be smooth. The large resistor $\mathrm{R}$ can offer protection when the load is light. The current of the large resistor $\mathrm{R}$ is about $10 \%$ of the minimum current output of the power. The output filter 
of front stage is $L_{f} C_{f}$ series filter. Since $C_{f}$ is usually an electrolytic capacitor, the low-frequency components of the input current of the latter stage can be filtered. Also, due to the filtering inductor, the influence which the latter stage input has on the front stage output will be reduced. The inductance is shown as follow ${ }^{[2]}$ :

$$
L_{f}=\frac{V_{o \text { max }} T_{o f f}}{I_{L f \max }}=\frac{V_{o \text { max }} T_{o f f}}{2 I_{o \text { min }}}
$$

$V_{o \max }$ is the maximum output dc voltage of front stage circuit, $T_{o f f}$ is the cut-off time of rectifier diode and $I_{o \text { min }}$ is the minimum output dc current of front stage circuit. Considering the output voltage ripple, the capacitance is like follow ${ }^{[3]}$ :

$$
C_{f}=\frac{V_{o \text { max }}(1-D) T_{s}^{2}}{8 L_{f} \Delta V_{o}}
$$

$\mathrm{D}$ is the output duty cycle of DC-DC circuit, $T_{s}$ is the switching cycles, and $\Delta V_{o}$ is the peak voltage of output voltage.

Figure 2 shows the traditional two-way asymmetric pulse circuit topology. By changing the output of voltage source $V_{1}$ in figure $2^{[4]}$, the positive pulse amplitude can be changed,

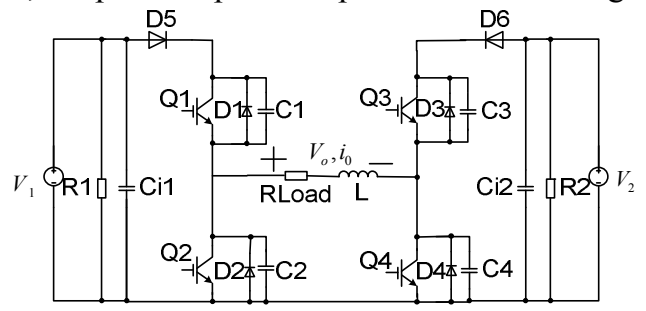

Figure 2. Traditional two-way asymmetric pulse circuit topology

In the same way, the negative pulse amplitude can be changed by changing the output of voltage source $V_{2}$. The voltage of load is $V_{1}$, when the switch device $Q_{1}$ and $Q_{4}$ is on. The voltage of load is $V_{2}$, when the switch device $Q_{3}$ and $Q_{2}$ is on. Since this topology requires two sets of voltage source, the cost and complexity of the power is high. This paper studies a new high-frequency asymmetric pulse power circuit topology ${ }^{[5]}$, the load is resistance and inductance load. Compared to the common full bridge topology, this topology reduces a switch $\left(Q_{2}\right)$.In figure 3(a), $I_{\text {in }}$ is an ideal current source, $R$ is a false load and $C$ is a filter capacitor. Using complementary PWM control mode. $Q_{1}$ and $Q_{4}$ are open at the same time, while $Q_{3}$ and $Q_{1}$ 、 $Q_{4}$ are opened complementary.

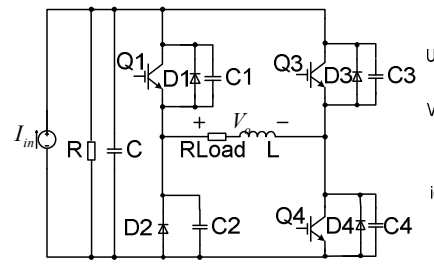

(a) Circuit topology

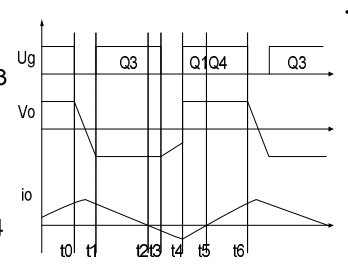

(b) Waveform diagram

Figure 3. A new type of high-frequency asymmetric pulse power circuit topology and waveform diagram

In figure $3(\mathrm{~b}), u_{g}$ is switch device drive waveform, $V_{o}$ is load voltage waveform and $i_{o}$ is load current waveform. The following assumptions are made:

(1) All switch devices and diodes are ideal device.

(2) All the capacitors and resistors are ideal device, besides the values of capacitance and resistance are large enough to reduce the value of the current flowing through the resistance. At the same time, capacitor voltage is the constant $V_{\text {in }}$.

(3) $C_{1}=C_{3}=C_{4}=\frac{4}{3} C_{\text {oss }}$

Converter has six working modes in one cycle. Switch mode 1: $Q_{1}$ and $Q_{4}$ are in the on-state till $t_{0} . V_{o}=V_{\text {in }}$, $i_{o}=i_{L \max }, u_{C 1}=u_{C 4}=0$, and $u_{C 2}=u_{C 3}=V_{\text {in }}$. At $t_{0}$, $Q_{1}$ and $Q_{4}$ are off. Because $L$ is large enough, $i_{o}=i_{L \max }$. Under the constant-current $i_{o}$, capacitor $C_{4}$ and $C_{1}$ are charged while capacitance $C_{2}$ and $C_{3}$ are discharged.

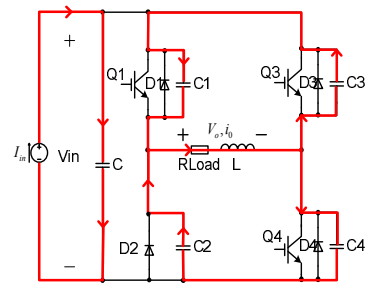

(a)Switch mode 1

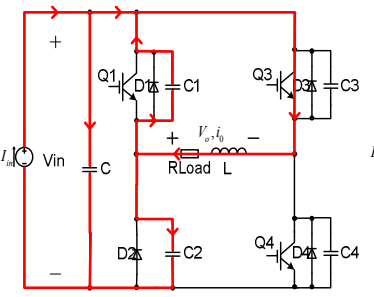

(c)Switch mode 3

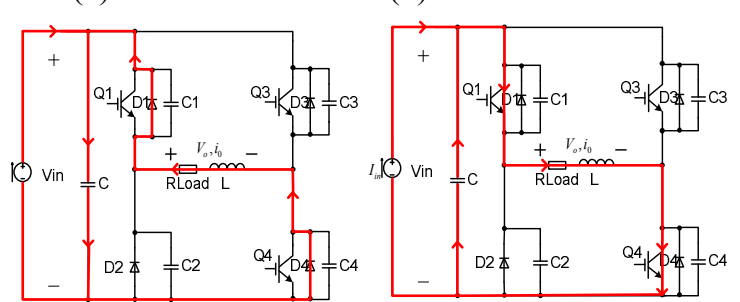

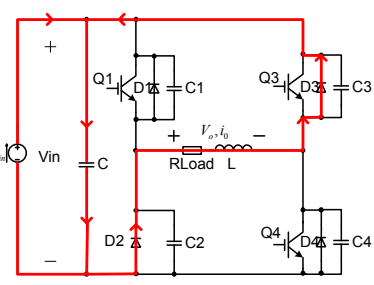

(b)Switch mode 2

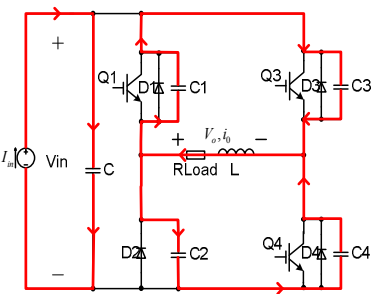

(d)Switch mode 4 
(e)Switch Mode5 $\quad$ (f) Switch Mode6

Figure 4. Equivalent circuit of converter in various modes

Switch mode 2:when $t=t_{1}, C_{1}$ and $C_{4}$ is charged to $\mathrm{V}_{\text {in }}$,

$C_{2}$ and $C_{3}$ is discharged to zero, the output voltage is $V_{o}=-V_{i n}$ and $u_{g 3}>0$.After loading the inductor freewheeling, switching tube $Q_{3}$ does not conduct, while diode $D_{2}$ and $D_{3}$ do. The equivalent circuit is shown in Figure 4(b).

Switch mode 3 : when $t=t_{2}, i_{o}=0$ and switching tube $Q_{3}$ zero-voltage is on. $C_{1}$ is discharged, $C_{2}$ is charged and $u_{C 2}=0$ 。

Switch mode 4: when $t=t_{3}$, switching tube $Q_{3}$ is off, and the inductor current approximately reached the minimum value $i_{L \min }, i_{o}=i_{L \min } . C_{2}$ and $C_{3}$ are charged with nearly constant current $i_{L \min }$ while $C_{1}$ and $C_{4}$ are discharged. Equivalent circuit is demonstrated in Figure 4(d).

Switch mode 5 : when $t=t_{4}, C_{2}$ and $C_{3}$ are charged to $V_{\text {in }}, C_{1}$ and $C_{4}$ are discharged to zero, the output voltage $V_{o}=V_{\text {in }}, u_{g 1}>0$ and $u_{g 4}>0$. After loading the inductor freewheeling, switching tube $Q_{1}$ and $Q_{4}$ are off and the body diode $D_{1}$ and $D_{4}$ are on, The equivalent circuit is shown in Figure 4(e) .

Switch mode 6: when $t=t_{5}$, load current is zero and switching tube $Q_{1}$ and $Q_{4}$ Zero-voltage is on. The equivalent circuit is shown in Figure 4(f). When $t=t_{6}$, switching tube $Q_{1}$ and $Q_{4}$ shutdown, coming back to modal 1 and it has come to the end of a work cycle

By the analyzing, during the switching $Q_{1}$ and $Q_{4}$ turn-off time, capacitor $C_{4}$ and $C_{1}$ are charged under the constant-current $i_{o}$, whereas capacitor $C_{2}$ and $C_{3}$ are discharged. And if the capacitor is large enough, there is still a certain voltage $V_{c 2}$ when discharge process is finished. As $Q_{3}$ conducts, the voltage of the load is $V_{i n}-V_{c 2}$ and the converter can output amplitude asymmetric negative pulse. The key is the selection of capacitor. In order to output amplitude asymmetric negative pulse, the selection of capacitor should make the resonant frequency $f_{0}$ less than pulse switching frequency $f$. The selection formula of capacitor is :

$$
f>f_{0}=\frac{1}{2 \pi \sqrt{L C_{2}}}
$$

If $f>f_{0}$, which means that capacitor $\mathrm{C}$ is very large in the case of constant inductor $\mathrm{L}$, then when tube $Q_{3}$ is open, the voltage of $C_{2}$ is $V_{\text {in }}$ and negative pulse output is zero. Otherwise, $C_{2}$ is very small. And when tube $Q_{3}$ is open, the voltage of $C_{2}$ is zero. Diode $D_{2}$ conducts and negative pulse output reaches maximum $V_{\text {in }}$.

\section{POWER CONTROL THEORY}

The later stage employs open-loop control, the front stage of DC/DC converter must be closed-loop control in order to make the input of the later stage stable. In this way the output voltage amplitude can be adjusted as required. Concerning the DC/DC converter, high-frequency asymmetric pulse converter is a special non-linear load. It directly affects the front stage DC/DC converter property. And it further affects the output results of the later stage converter. The high-frequency asymmetric pulse power of current source block diagram is shown in Figure 1.

In a cycle of the later stage converter, when the switching device is turned off, capacitor $\mathrm{C}$ is charged with the constant current $I_{L}$, When the later stage switching device is turned on, capacitor $\mathrm{C}$ is discharged to the load. At this time the load current is equal to the sum of the DC/DC converter constant current output $I_{L}$ and the capacitor discharge current. During a cycle, the average current through the capacitor should be zero. If the average current consumed by the false load $\mathrm{R}$ is ignored, the average current $I_{\text {Load }}$ of flowing through the load is equal to the front stage DC/DC constant output current $I_{L}$.Therefore, the later stage load current can be constant by keeping the front stage 's output current constant. Therefore, as long as the front stage current is constant, the average current flowing through the load will not change. However the voltage of the capacitor will change with the duty cycle of the later stage converter. The greater the duty cycle, the shorter the capacitor's charging time. Also, the discharge time will be longer and as a result, the capacitor voltage will be lower; on the contrary, the smaller the duty cycle, the longer the charging time for the capacitor, the shorter the discharge time and the higher the capacitor voltage.

\section{EXPERIMENT RESULTS AND ANALYSIS}

A $1 \mathrm{~kW}$ experimental prototype of which the pulse frequency is $20 \mathrm{kHz} \sim 50 \mathrm{kHz}$ was built to verify the circuit principle. The DC/DC converter of the front stage is the current model and the current can be regulated from $0.3 \mathrm{~A}$ to 1A. Filtering inductor $L_{f}=8 m H$. capacitor $C_{f}=10 u F$, the false load $R=10 k \Omega$ and the filter capacitor is the high-frequency with no-sense capacitance 
$C_{f}=1 u F$. Switch device FGA25N120ANTD is used in the later stage. $C_{1}=C_{3}=C_{4}=1 n F . C_{2}=10 n F$. Diode DSEI12-12A is used and switch frequency is $50 \mathrm{kHz}$. Dead time is $1 \mu \mathrm{s}$. Resistance and inductance load are adopted, of which the value is $50 \Omega$ and $1.55 \mathrm{mH}$ respectively. UC3843 and UC3715 are used to make the open loop control in the later stage. Figure 5 and Figure 6 are the voltage and current waveforms of the load. The duty cycle is $10 \%$ in Figure 5 while it is $80 \%$ in Figure 6.

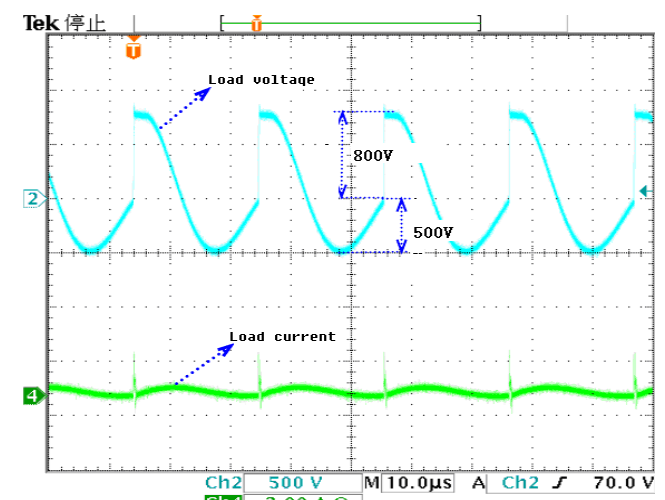

Figure 5. Voltage and current waveforms, the duty cycle is $10 \%$

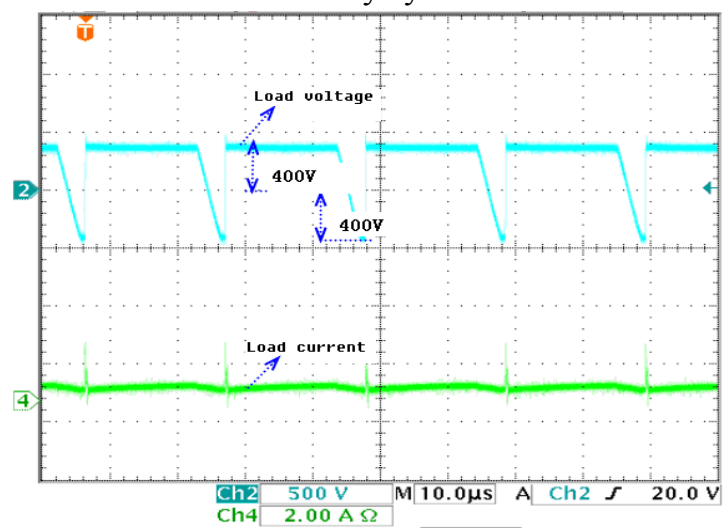

Figure 6. Voltage and current waveforms, the duty cycle is $80 \%$
By the figure, voltage amplitude of the load is very stable and it meets the control requirements. Experimental waveforms are almost the same with theoretical analysis waveforms. The positive and negative amplitude of output voltage can be adjusted and the pulse duty cycle can also be adjusted in a certain range. The circuit can meet the requirements of high-frequency asymmetric pulse power.

\section{CONCLUSION}

This paper has presented a kind of new high-frequency asymmetric pulse power current source topology and its control method. Both positive and negative asymmetric pulse voltage can be produced and the amplitude of the pulse can be adjusted. While the dutycycle of the positive pulses can be adjusted from $10 \%$ to $80 \%$, the frequency of the pulse ranges from $20 \mathrm{kHz}$ to $50 \mathrm{kHz}$. Theoretical analysis has been validated by the experiment results. The topology, which has the advantages of simple structure, easy to control and low cost, is considered to be suitable for the surface treatment of material.

[1] Ren Xiaoyong, Ruan xinbo. Two-Stage converter applicable to high voltage input low voltage output case[J].Proceeding of the CSEE,2005,25 (23) :154-156

[2] Ruan Xinbo, Yan Yangguang. Soft-switch technology of DC switching power supply[M].Science press. 2000:101

[3] Jesus Doval-Gandoy,Carlos Castro.Line Input AC-to-DC Conversion and Filter Capacitor Design[J],IEEE Transactions on Industry Applications, 2003,39(4): 1169-1176

[4] Yang shiyan, Bi hongqi. Two-way asymmetric pulse power realization circuit design[J] .Power Electronics, 2003.3:16-17

[5] Lin Weixun. Modern power electronics [M].Mechanical industry press, 2005,4:402-406 